\title{
Representation dimension of artin algebras
}

\section{Steffen Oppermann}

Institutt for matematiske fag

NTNU

7491 Trondheim

Norway

E-mail address: Steffen.Oppermann@math.ntnu.no

In 1971, Auslander [1] has introduced the notion of representation dimension of an artin algebra. His definition is as follows (see Section 1 for details on the notation):

Definition A. Let $\Lambda$ be an artin algebra. We set

$A(\Lambda)=\left\{\Gamma\right.$ artin algebra $\mid$ dom $\cdot \operatorname{dim} \Gamma \geq 2$ and $\left.\Lambda \underset{\text { Morita }}{\sim} \operatorname{End}_{\Gamma}\left(I_{0}(\Gamma)\right)\right\}$.

Here $I_{0}(\Gamma)$ denotes the injective envelope of $\Gamma$ as $\Gamma$-module.

The representation dimension of $\Lambda$ is defined as

$$
\operatorname{repdim} \Lambda= \begin{cases}1 & \text { if } \Lambda \text { is semi-simple } \\ \min \{\mathrm{gl} \cdot \operatorname{dim} \Gamma \mid \Gamma \in A(\Lambda)\} & \text { otherwise }\end{cases}
$$

Auslander has shown that an algebra is of finite representation type, that is, it admits only finitely many indecomposable modules up to isomorphism, if and only if its representation dimension is at most 2 . We will give a proof of this fact in Section 1 as Corollary 1.9. This led Auslander to the expectation, "that this notion gives a reasonable way of measuring how far an artin algebra is from being of finite representation type." [1, III.5, lines 2,3$]$

Igusa and Todorov [6] have shown that there is a connection between Auslander's representation dimension and the finitistic dimension conjecture.

Finitistic dimension conjecture. Let $\Lambda$ be an artin algebra, and fin.dim $\Lambda=\sup \{\operatorname{pd} X \mid X \in \bmod \Lambda$ such that $\operatorname{pd} X<\infty\}$. 
Then

$$
\text { fin. } \operatorname{dim} \Lambda<\infty \text {. }
$$

More precisely, they have shown that the finitistic dimension conjecture holds for any artin algebra of representation dimension at most three. Here we will prove this in Section 2.

Unfortunately, it turned out to be rather hard to compute the actual value of the representation dimension of a given algebra. However, in 2003 Iyama [8] has shown that the representation dimension of a finite dimensional algebra is always finite. He did so by explicitly constructing an algebra $\Gamma \in A(\Lambda)$ with gl.dim $\Gamma<\infty$, so the minimum in the definition above is always finite. We will explain his construction and prove that it works in Section 3 here. When applied to a given algebra, this construction yields an upper bound for the representation dimension of this algebra.

By Auslander's result mentioned above, it was known that any representation infinite algebra has representation dimension at least three. However, it was not known whether numbers greater than three can occur as the representation dimension of a finite dimensional algebra, until Rouquier [12] has shown in 2005 that the representation dimension of the exterior algebra of an $n$-dimensional vector space is always $n+1$. Here, in Section 4, we will take a slightly different approach to show that any number occurs as the representation dimension of some artin algebra. In order to do so we will give Rouquier's definition of dimension of a triangulated category [12, 13], and the author's generalization to subcategories [10]. We will then explain what is the connection of these dimensions to Auslander's representation dimension. Finally we will use this method to determine the representation dimension of the Beilinson algebras.

\section{Different definitions and first properties}

In this section we give three different definitions of representation dimension. We show that, provided the algebra is not semi-simple, all three definitions are equivalent. We will give some indication as to when which definition is most helpful. In particular we will prove Auslander's theorem, saying that an algebra has representation dimension at most two if and only if it is representation finite, using Definition C. 
Notation. For an artin algebra $\Lambda$ we denote by $\bmod \Lambda$ the category of finitely generated left $\Lambda$-modules. We denote by $D: \bmod \Lambda \rightarrow \bmod \Lambda^{\text {op }}$ the standard duality.

Let us start by recalling Auslander's original definition as given in the introduction. First we define the notions involved.

Notation. Let $\Lambda$ be an artin algebra. Let $M \in \bmod \Lambda$. Then we denote by pd $M$ and id $M$ the projective dimension and injective dimension of $M$, respectively. We denote by gl.dim $\Lambda$ the global dimension of $\Lambda$, that is the maximum over the projective dimensions of all modules.

1.1. Definition. Let $\Lambda$ be an artin algebra, and let

$$
\Lambda \longrightarrow I_{0}(\Lambda) \longrightarrow I_{1}(\Lambda) \longrightarrow \cdots
$$

be a minimal injective resolution. Then the dominant dimension of $\Lambda$ is

$$
\text { dom.dim } \Lambda=\inf \left\{n \in \mathbb{N} \mid I_{n}(\Lambda) \text { is not projective }\right\} .
$$

1.2. Definition. Let $\Lambda$ and $\Gamma$ be to artin algebras. We say $\Lambda$ and $\Gamma$ are Morita equivalent (denoted by $\Lambda \underset{\text { Morita }}{\sim} \Gamma$ if there is a projective generator $P \in \bmod \Lambda$ (that is a projective module $P \in \operatorname{proj} \Lambda$ such that $\Lambda \in$ add $P)$ such that $\Gamma \cong \operatorname{End}_{\Lambda}(P)$.

Definition A. Let $\Lambda$ be an artin algebra. We set

$A(\Lambda)=\left\{\Gamma\right.$ artin algebra $\mid$ dom $\cdot \operatorname{dim} \Gamma \geq 2$ and $\left.\Lambda \underset{\text { Morita }}{\sim} \operatorname{End}_{\Gamma}\left(I_{0}(\Gamma)\right)\right\}$.

Here $I_{0}(\Gamma)$ denotes the injective envelope of $\Gamma$ as $\Gamma$-module.

The representation dimension of $\Lambda$ is defined as

$$
\operatorname{repdim} \Lambda= \begin{cases}1 & \text { if } \Lambda \text { is semi-simple } \\ \min \{\operatorname{gl} \cdot \operatorname{dim} \Gamma \mid \Gamma \in A(\Lambda)\} & \text { otherwise. }\end{cases}
$$

1.3. Observations. - The condition $\operatorname{dom} \cdot \operatorname{dim} \Gamma \geq 1$ means that (up to multiplicity) $I_{0}(\Gamma)$ is the direct sum of all indecomposable projective injective $\Lambda$-modules.

- We set

$$
\begin{gathered}
\mathcal{S}=\{X \in \bmod \Gamma \mid \text { there is an exact sequence } \\
\qquad \longmapsto B_{0} \longrightarrow B_{1} \text { with } \\
\left.B_{0}, B_{1} \in \text { add } I_{0}(\Gamma)\right\} .
\end{gathered}
$$


Since $I_{0}(\Gamma)$ is injective the functor $\mathrm{F}: \mathcal{S} \rightarrow \bmod \Lambda$ as depicted in the following diagram is an equivalence.

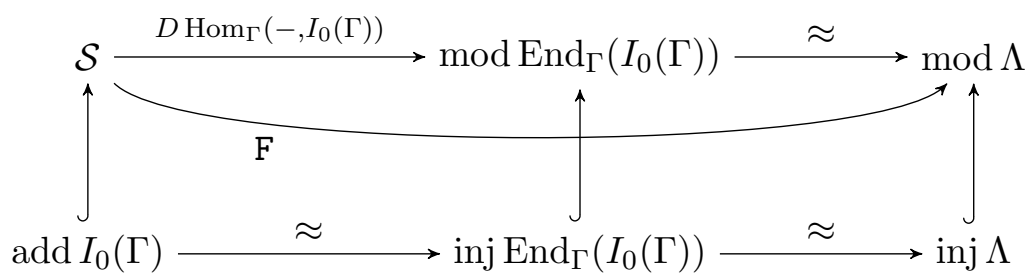

- Since dom.dim $\Gamma \geq 2$ we see that $\Gamma \in \mathcal{S}$. In particular it makes sense to consider $F(\Gamma)$, and

$$
\operatorname{End}_{\Lambda}(F(\Gamma))=\operatorname{End}_{\Gamma}(\Gamma)=\Gamma \text {. }
$$

- The $\Lambda$-module $F(\Gamma)$ is a generator and cogenerator of $\bmod \Lambda$. That is, it contains all indecomposable projective and all indecomposable injective $\Lambda$ modules as direct summands.

This motivates the following version of Auslander's original definition.

Definition B. Let $\Lambda$ be an artin algebra. Then

$$
\begin{array}{r}
\operatorname{repdim} \Lambda=\min \{\text { gl.dim } \operatorname{End}(M) \mid M \in \bmod \Lambda \\
\text { generator and cogenerator }\} .
\end{array}
$$

A generator cogenerator $M$ realizing the minimum above is called Auslander generator.

We have seen above that any algebra in $A(\Lambda)$ is the endomorphism ring of a generator cogenerator of $\bmod \Lambda$. Hence, in order to show that the two definitions coincide for any non-semisimple algebra $\Lambda$, it suffices to show than $\operatorname{End}_{\Lambda}(M) \in A(\Lambda)$ for any generator cogenerator $M$ of $\bmod \Lambda$.

Proof. Let $M \longmapsto I_{0}(M) \rightarrow I_{1}(M)$ be the start of an injective resolution of $M$. Applying the functor $\operatorname{Hom}(M,-)$ we obtain

$$
\operatorname{End}_{\Lambda}(M) \longrightarrow \operatorname{Hom}_{\Lambda}\left(M, I_{0}(M)\right) \longrightarrow \operatorname{Hom}_{\Lambda}\left(M, I_{1}(M)\right) \text {. }
$$

Now $\operatorname{Hom}_{\Lambda}(M, D \Lambda)=D \operatorname{Hom}_{\Lambda}(\Lambda, M)$, and this is injective as $\operatorname{End}_{\Lambda}(M)$-module since $\Lambda \in$ add $M$. Hence the sequence above shows 
that

dom.dim $\operatorname{End}_{\Lambda}(M) \geq 2$, and moreover that

$$
\begin{gathered}
\operatorname{End}_{\operatorname{End}_{\Lambda}(M)}\left(I_{0}\left(\operatorname{End}_{\Lambda}(M)\right)\right)=\operatorname{End}_{\operatorname{End}_{\Lambda}(M)}\left(\operatorname{Hom}_{\Lambda}\left(M, I_{0}(M)\right)\right) \\
=\operatorname{End}_{\Lambda}\left(I_{0}(M)\right) \underset{\text { Morita }}{\sim} \Lambda .
\end{gathered}
$$

Here the Morita equivalence follows from the fact that $M$ is a cogenerator.

1.4. Definition. For $M \in \bmod \Lambda$ and $X \in \bmod \Lambda$ we say that $f: M^{\prime} \rightarrow X$ is a right $M$-approximation, if any morphism $\varphi: M \rightarrow X$ factors through $f$.

It is called minimal right $M$-approximation, if it is moreover right minimal in the sense of [3].

We denote by $\Omega_{M} X$ the kernel of a minimal right $M$-approximation of $X$, and inductively we set $\Omega_{M}^{n+1} X=\Omega_{M} \Omega_{M}^{n} X$.

We set

$$
\begin{aligned}
M \text {-resol.dim } X & =\inf \left\{n \in \mathbb{N} \mid \Omega_{M}^{n+1} X=0\right\}, \text { and } \\
M \text {-resol.dim }(\bmod \Lambda) & =\sup \{M \text {-resol.dim } X \mid X \in \bmod \Lambda\} .
\end{aligned}
$$

1.5. Observations. - The $M$-approximations and the derived constructions only depend on add $M$.

- For $M=\Lambda$ the $M$-approximations are projective covers, and $\Omega_{\Lambda}=\Omega$ is the usual syzygy.

- If $\Lambda \in$ add $M$ then right $M$-approximations are always epimorphisms.

- The functor

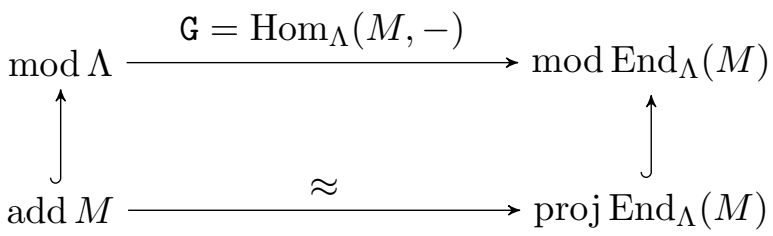

maps

- kernels to kernels, and

- right $M$-approximations to projective covers.

In particular for any $X \in \bmod \Lambda$ we have $M$-resol.dim $X=$ $\operatorname{pd} \mathrm{G}(X)$. 
- For any $X \in \bmod \Lambda$ we there is a sequence

$$
\Omega_{M}^{2} X \longrightarrow M_{1} \longrightarrow M_{0} \longrightarrow X
$$

which is exact in $M_{1}$. Therefore $\Omega_{M}^{2} X \in \operatorname{Im}$ G.

- For $X \in \bmod \Lambda$ we have $\mathrm{G}(X) \in \Omega^{2}(\bmod \Gamma)$ if and only if there is an exact sequence $X \leadsto M^{0} \rightarrow M^{1}$ with $M^{0}, M^{1} \in$ add $M$.

- If $M$ is a cogenerator then we have $\mathrm{G}(X) \in \Omega^{2}(\bmod \Gamma)$ for any $X \in \bmod \Lambda$.

Hence we have shown that Definition B is equivalent to the following:

Definition C. Let $\Lambda$ be an artin algebra. Then

$$
\begin{array}{r}
\text { repdim } \Lambda=\min \{M \text {-resol.dim }(\bmod \Lambda) \mid M \in \bmod \Lambda \\
\text { generator and cogenerator }\}+2 .
\end{array}
$$

1.6. Remark. Motivated by this definition and the examples of algebras where the representation dimension is explicitly known (see Theorems 4.14 and 4.15) Ringel suggests to normalize the representation dimension, and set

$$
\text { n.repdim } \Lambda=\operatorname{repdim} \Lambda-2 \text {. }
$$

Let us summarize what we have shown so far.

1.7. Theorem. Let $\Lambda$ be an artin algebra, which is not semi-simple. Then Definitions $A, B$, and $C$ are equivalent. (It is easy to see that they give 1, 0, and 2, respectively for semi-simple algebras.)

1.8. Remarks. - One advantage of Definition B is, that to use it we have to know only $M$, and not the rest of the module category.

- One advantage of Definition $\mathrm{C}$ is that, if we do not know $M$, we can work in the module category of $\Lambda$ and don't have to construct projective resolutions in an unknown module category.

1.9. Corollary (Auslander). Let $\Lambda$ be an artin algebra. Then repdim $\Lambda$ $\leq 2$ if and only if $\Lambda$ has finite representation type.

Proof. Assume first that $\Lambda$ has finite representation type. Then we can find an additive generator $M$ of $\bmod \Lambda$ (that is a module $M \in \bmod \Lambda$, such that add $M=\bmod \Lambda$ ). One easily sees that $M$-resol.dim $X=0$ for any $X \in \bmod \Lambda$, and hence $\operatorname{repdim} \Lambda=2$. 
Now assume that $\Lambda$ is not representation finite, and let $M$ be an Auslander generator. Then there is $X \in \bmod \Lambda \backslash$ add $M$. For this $X$ we have $M$-resol.dim $X \geq 1$, and hence $M$-resol.dim( $\bmod \Lambda) \geq 1$. Therefore repdim $\Lambda \geq 3$.

1.10. Example. Let $\Lambda$ be the quiver algebra of linearly oriented $A_{4}$, with Auslander-Reiten quiver as depicted below.

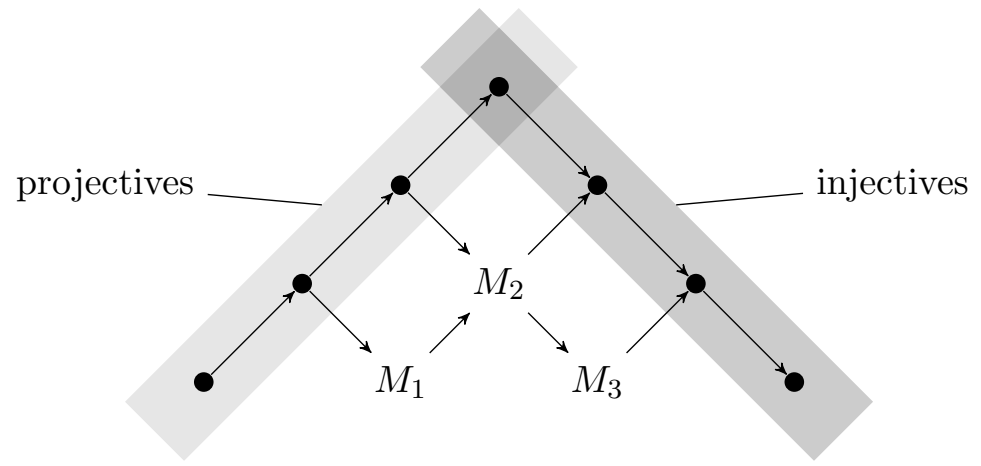

We obtain the following resolution dimensions:

\begin{tabular}{l|c}
$M$ & $M$-resol.dim $(\bmod \Lambda)$ \\
\hline$\Lambda \oplus D \Lambda$ & 1 \\
$\Lambda \oplus D \Lambda \oplus M_{1}$ & 1 \\
$\Lambda \oplus D \Lambda \oplus M_{2}$ & 2 \\
$\Lambda \oplus D \Lambda \oplus M_{3}$ & 1 \\
$\Lambda \oplus D \Lambda \oplus M_{1} \oplus M_{2}$ & 1 \\
$\Lambda \oplus D \Lambda \oplus M_{1} \oplus M_{3}$ & 1 \\
$\Lambda \oplus D \Lambda \oplus M_{2} \oplus M_{3}$ & 1 \\
$\Lambda \oplus D \Lambda \oplus M_{1} \oplus M_{2} \oplus M_{3}$ & 0
\end{tabular}

This suggests that the $M$-resultion dimension tends to go down if we make $M$ bigger. However this is not true in general, and in fact we have

1.11. Theorem (Iyama [7, Theorem 4.6.2]). Let $\Lambda$ be controlled wild, and $n \in \mathbb{N}$. Then

$$
\begin{gathered}
\exists B: \forall M \text { generator and cognerator with } B \in \text { add } M: \\
M \text {-resol.dim }(\bmod \Lambda) \geq n
\end{gathered}
$$

We now follow Ringel [11] to determine a large class of algebras which have representation dimension three. 
1.12. Theorem. Assume the category

$$
\operatorname{Sub} \Lambda=\left\{X \in \bmod \Lambda \mid X \hookrightarrow \Lambda^{n} \text { for some } n\right\}
$$

contains only finitely many indecomposables (such an algebra is called torsionless finite). Then repdim $\Lambda \leq 3$. In particular, any representation infinite torsionless finite algebra has representation dimension three.

Proof. See [11] for the proof that a torsionless finite algebra is also cotorsionless finite, that is the category

$$
\text { Fac } D \Lambda=\left\{X \in \text { ind } \Lambda \mid D \Lambda^{n} \rightarrow X \text { for some } n\right\}
$$

also contains only finitely many indecomposables.

We choose $\underline{M}$ and $\bar{M}$ such that $\operatorname{add} \underline{M}=\operatorname{Sub} \Lambda$ and add $\bar{M}=$ Fac $D \Lambda$, and set $M=\underline{M} \oplus \bar{M}$.

Let $X \in \bmod \Lambda$. Let $\underline{M}^{\prime} \stackrel{f}{\longrightarrow} X$ and $\bar{M}^{\prime} \stackrel{\bar{f}}{\longrightarrow} X$ be minimal right $\underline{M}$ - and $\bar{M}$-approximations of $X$. Since $\Lambda \in \operatorname{add} \underline{M}$ the map $f$ is onto. Since add $\bar{M}$ is closed under factors $\bar{f}$ is into. We take the pullback as in the following diagram.

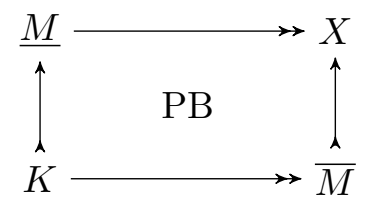

Now, since add $\underline{M}$ is closed under subobjects we have $K \in \operatorname{add} \underline{M}$, and hence an $M$-resolution

$$
K \longrightarrow \underline{M^{\prime}} \oplus \bar{M}^{\prime} \longrightarrow X .
$$

Hence $M$-resol.dim $(\bmod \Lambda) \leq 1$, and the claim follows from Definition $\mathrm{C}$.

1.13. Examples. The following classes of algebras are torsionless finite, and hence have representation dimension at most three.

- hereditary algebras

- concealed algebras

- algebras with $\operatorname{Rad}^{2}=0$ 


\section{The connection to the finitistic dimension conjecture}

In this section we summarize Igusa and Tororov's proof of the following result.

2.1. Theorem (Igusa-Todorov [6]). Let $\Lambda$ be an artin algebra of representation dimension at most three. Then

$$
\text { fin. } \operatorname{dim} \Lambda<\infty \text {. }
$$

Recall that fin. $\operatorname{dim} \Lambda=\sup \{\operatorname{pd} X \mid X \in \bmod \Lambda \wedge \operatorname{pd} X<\infty\}$. One of the most important conjectures about homological properties of artin algebras is the following:

Finitistic dimension conjecture. Let $\Lambda$ be an artin algebra, and

$$
\text { fin.dim } \Lambda=\sup \{\operatorname{pd} X \mid X \in \bmod \Lambda \text { such that } \operatorname{pd} X<\infty\} .
$$

Then

$$
\text { fin. } \operatorname{dim} \Lambda<\infty \text {. }
$$

Hence Igusa and Todorov's theorem says that the finitistic dimension conjecture holds for algebras of representation dimension at most three.

2.2. Remark. It can be very easy to show that repdim $\Lambda \leq 3$ (using Definition B): If we have a generator cogenerator $M$ it is easy to check if gl.dim $\operatorname{End}(M) \leq 3$.

Idea of proof of 2.1. Let $M$ be an Auslander generator. We have to find an upper bound to $\operatorname{pd} X$ for any $X \in \bmod \Lambda$ of finite projective dimension.

By Definition $\mathrm{C}$ and the assumption of the theorem there is a short exact sequence

$$
M_{1} \longmapsto M_{0} \longrightarrow X
$$

with $M_{1}$ and $M_{0} \in$ add $M$. Hence there are also short exact sequences

$$
\Omega^{n} M_{1} \longrightarrow \Omega^{n} M_{0} \oplus P \longrightarrow \Omega^{n} X
$$

for any $n$ (with $P$ projective). Since $X$ has finite projective dimension eventually we will have

$$
\Omega^{n} M_{1} \cong \Omega^{n} M_{0}
$$


Now Igusa and Todorov apply the fitting lemma to show that there is $\phi(M) \in \mathbb{N}$ such that for any $M^{\prime}, M^{\prime \prime} \in \operatorname{add} M$

$$
\begin{aligned}
& \Omega^{n} M^{\prime} \cong \Omega^{n} M^{\prime \prime} \text { for some } n \in \mathbb{N} \\
\Longrightarrow & \Omega^{\phi(M)} M^{\prime} \cong \Omega^{\phi(M)} M^{\prime \prime}
\end{aligned}
$$

(see [6] for details).

Now split $\Omega^{\phi(M)} M_{0}=\Omega^{\phi(M)} M_{1}=A \oplus B$, with $\operatorname{pd} A<\infty$ and $B$ a direct sum of indecomposable modules of infinite projective dimension. In the short exact sequence

$$
A \oplus B \stackrel{\left(\begin{array}{ll}
* & * \\
* & f_{B} \\
* & *
\end{array}\right)}{\longrightarrow} A \oplus B \oplus P \longrightarrow \Omega^{\phi(M)} X
$$

the map $f_{B}$ has to be an isomorphism (see $[6]$ ). Therefore there is a short exact sequence

$$
A \longmapsto A \oplus P \longrightarrow \Omega^{\phi(M)} X .
$$

Hence

$$
\begin{aligned}
\operatorname{pd} X & \leq \phi(M)+\operatorname{pd} A+1 \\
& \leq \phi(M)+\max \left\{\operatorname{pd} X \mid X \in \operatorname{add} \Omega^{\phi(M)} M \text { and } \operatorname{pd} X<\infty\right\}+1 .
\end{aligned}
$$

Since this upper bound for pd $X$ does not depend on $X$ it follows that fin. $\operatorname{dim} \Lambda \leq \phi(M)+\max \left\{\operatorname{pd} X \mid X \in \operatorname{add} \Omega^{\phi(M)} M\right.$ and $\left.\operatorname{pd} X<\infty\right\}+1$.

2.3. Example. Erdmann, Holm, Iyama, and Schröer [5] have shown that any special biserial algebra has representation dimension at most three. With Theorem 2.1 they obtain as a corollary that any special biserial algebra has finite finitistic dimension.

2.4. Remarks. - The proof only requires the existence of a short exact sequence $M_{1} \leadsto M_{0} \rightarrow X$ for any $X$, but does not need this sequence to be an $M$-resolution, or $M$ to be a cogenerator.

- In order to show that the finitistic dimension is finite, it would suffice to treat all $X$ which are syzygies. 


\section{Iyama's finiteness theorem}

In this section we give a proof of the following result of Iyama.

3.1. Theorem (Iyama $[7,8])$. Let $\Lambda$ be an artin algebra. Then

repdim $\Lambda<\infty$.

The strategy of the proof is to construct a generator cogenerator $M \in \bmod \Lambda$ such that $M$-resol.dim $(\bmod \Lambda)<\infty$.

3.2. Remark. Iyama [8] has first shown that the generator cogenerator $M$ he constructed has quasi-hereditary endomorphism ring. Hence its endomorphism ring has finite global dimension, and the representation dimension is finite by Definition B.

3.3. Construction. Let $M^{0}$ be any generator cogenerator (typically we choose $\left.M^{0}=\Lambda \oplus D \Lambda\right)$. Then set inductively

$$
M^{i+1}=M^{i} / \operatorname{Soc}_{E_{n}\left(M^{i}\right)} M^{i} .
$$

That means $M^{i+1}$ is the image of the map

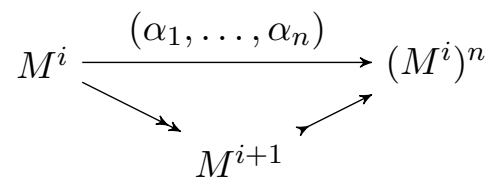

where $\alpha_{1}, \ldots, \alpha_{n}$ generate $\operatorname{Rad}\left(M^{i}, M^{i}\right)_{\operatorname{End}\left(M^{i}\right)}$, that is any radical endomorphism of $M^{i}$ factors through $\left(\alpha_{1}, \ldots, \alpha_{n}\right)$. In particular the $M^{i}$ have the properties

(1) Any radical map $M^{i} \rightarrow M^{i}$ factors through $M^{i} \stackrel{\text { proj }}{\longrightarrow} M^{i+1}$.

(2) For any $i$ we have $\operatorname{Sub} M^{i+1} \subseteq \operatorname{Sub} M^{i}$.

Note that whenever $M^{i} \neq 0$ we have $\operatorname{length}\left(M^{i+1}\right) \lesseqgtr \operatorname{length}\left(M^{i}\right)$. Since $M^{0}$ has finite length there is $m$ such that $M^{m+1}=0$. We set

$$
M=\oplus_{i=0}^{m} M^{i} .
$$

Clearly this is a generator cogenerator.

3.4. Proposition. With $M$ and $m$ as above we have

$$
M \text {-resol.dim }(\bmod \Lambda) \leq m-1 \text {. }
$$


In particular

$$
\operatorname{repdim} \Lambda \leq m+1
$$

Clearly this implies Theorem 3.1.

Proof. Let $X \in \bmod \Lambda$ not contain any injective direct summands. Then we show the following by induction:

$\left(A_{k}\right)$ There is a radical monomorphism $\Omega_{M}^{k} X \longrightarrow\left(M^{k}\right)^{n}$ for some $n$.

$\left(B_{k}\right)$ There is an $M$-approximation $M^{\prime} \rightarrow \Omega_{M}^{k} X$ with $M^{\prime} \in$ add $\oplus_{i=k+1}^{m} M^{i}$.

$\left(A_{0}\right)$ is true (take an injective envelope). We will show $\left(A_{k}\right) \underset{(2)}{\Longrightarrow}\left(B_{k}\right)$ $\underset{(1)}{\Longrightarrow}\left(A_{k+1}\right)$.

For implication (1) note that, in the setup of $\left(B_{k}\right)$, we have

$$
\Omega_{M}^{k+1} X \stackrel{\in \operatorname{Rad}}{\longrightarrow} M^{\prime} \longmapsto\left(M^{k+1}\right)^{n}
$$

for some $n$ by Property (2) in Construction 3.3 above.

For implication (2) let $s$ be the biggest number such that there is an $M$-approximation $M^{\prime} \rightarrow \Omega_{M}^{k}$ with $M^{\prime} \in \operatorname{add} \oplus_{i=s+1}^{m} M^{i}$. We may assume $M^{\prime}$ to be of the form $\oplus_{i=s+1}^{m}\left(M^{i}\right)^{n_{i}}$ for some $n_{i}$. If we assume $s<k$ we have the following diagram,

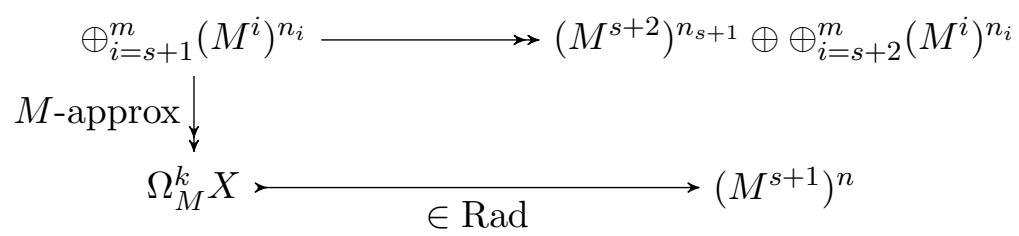

where the upper map is projection in the components $M^{s+1} \rightarrow M^{s+2}$ and identity elsewhere. By Property (1) of Construction 3.3 we can complete this to a commutative square. Since the image of the right vertical map is the same as the image of the composition from left upper to right lower corner, which is $\Omega_{M}^{k} X$, we obtain the following 
commutative diagram.

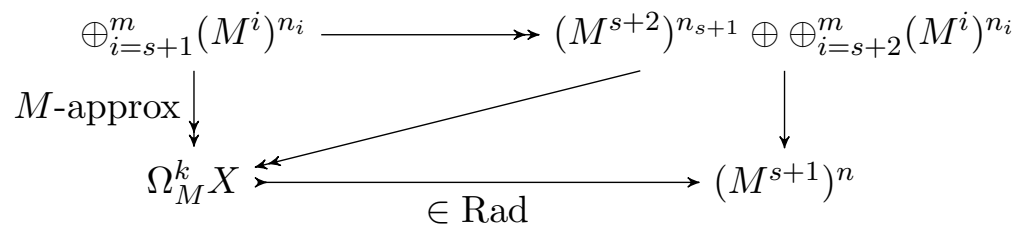

Now the diagonal map is also an $M$-approximation, contradicting the minimality of $s$. Hence $s \geq k$, and the inductive statements are shown.

Finally we see that by $\left(B_{m}\right)$ we have $\Omega_{M}^{m} X=0$, and hence $M$-resol.dim $X \leq m-1$.

3.5. Example (Result by Auslander). Let $\Lambda$ with LL $\Lambda=2$ (LL denotes the Loewy length).

$$
\begin{array}{ll}
M^{0}=P \oplus D \Lambda & \quad(P \text { projective non-injective }) \\
M^{1}=P \oplus \underbrace{D \Lambda / \operatorname{Soc} D \Lambda}_{\text {semisimple }} & \\
M^{2}=\text { semisimple } & \\
M^{3}=0 & \Longrightarrow \operatorname{repdim} \Lambda \leq 3 .
\end{array}
$$

3.6. Example (Result by Auslander). Let $\Lambda$ be self-injective. $M^{0}=$ $\Lambda$. Inductively one sees that the indecomposable direct summands of $M^{i}$ have pairwise non-isomorphic simple tops, and hence LL $M^{i+1}<$ LL $M^{i}$. Therefore $M^{\mathrm{LL} \Lambda}=0$, and $\operatorname{repdim} \Lambda \leq \operatorname{LL} \Lambda$.

3.7. Example (Iyama). Let $\Lambda$ be the Beilinson-algebra $k Q / I$ with

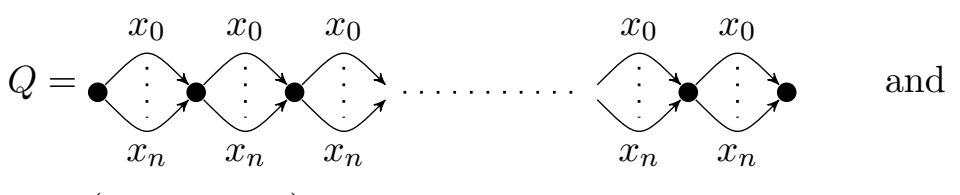

$$
I=\left(x_{i} x_{j}-x_{j} x_{i}\right) .
$$

Starting with $M^{0}=\Lambda \oplus D \Lambda$ one obtains $M^{n+2}=0$, and hence $\operatorname{repdim} \Lambda \leq n+2$. 


\section{Lower bounds}

In this section we will restrict ourselves to finite dimensional $k$ algebras.

4.1. Idea (Rouquier). $M$-resol.dim $X$ big $\approx$ it takes many short exact sequences to build $X$ from $M$.

Translation to the triangulated world gives the dimension of a (subcategory of a) triangulated category.

4.2. Construction. Let $\mathcal{T}$ be a triangulated category, and $M \in \mathcal{T}$.

$$
\begin{gathered}
\langle M\rangle=\langle M\rangle_{1}=\operatorname{add}\{M[i] \mid i \in \mathbb{Z}\} \\
\langle M\rangle_{n+1}=\operatorname{add}\left\{X \mid \exists M^{\prime} \rightarrow X \rightarrow M^{\prime \prime} \rightarrow M^{\prime}[1]\right. \text { with } \\
\left.M^{\prime} \in\langle M\rangle, M^{\prime \prime} \in\langle M\rangle_{n}\right\}
\end{gathered}
$$

The subcategory $\langle M\rangle_{n}$ is also called " $n^{\text {th }}$ thickening of $M$ ". It contains all objects that can be constructed from $M$ using triangles at most $n-1$ times.

4.3. Definition. The dimension of a triangulated category $\mathcal{T}$ is

$$
\operatorname{dim} \mathcal{T}=\inf \left\{n \mid \exists M \in \mathcal{T}: \mathcal{T}=\langle M\rangle_{n+1}\right\} .
$$

For a subcategory $\mathcal{C} \subseteq \mathcal{T}$ the dimension is defined to be

$$
\operatorname{dim}_{\mathcal{T}} \mathcal{C}=\inf \left\{n \mid \exists M \in \mathcal{T}: \mathcal{C} \subseteq\langle M\rangle_{n+1}\right\} .
$$

We will omit the index $\mathcal{T}$ when there is no danger of confusion.

Of particular interest to us are $\operatorname{dim} D^{b}(\bmod \Lambda)$ and $\operatorname{dim} \bmod \Lambda=$ $\operatorname{dim}_{D^{b}(\bmod \Lambda)} \bmod \Lambda$.

4.4. Lemma. Let $M \in \bmod \Lambda$ be a generator, and $X \in \bmod \Lambda$. Then for any $n$ we have

$$
M \text {-resol.dim } X \leq n \Longrightarrow X \in\langle M\rangle_{n+1} .
$$

In particular

$$
\begin{aligned}
M-\text { resol.dim }(\bmod \Lambda) & \geq \operatorname{dim} \bmod \Lambda, \quad \text { and } \\
\operatorname{repdim} \Lambda & \geq \operatorname{dim} \bmod \Lambda+2 .
\end{aligned}
$$

Proof. This follows immediately from the fact that short exact sequences in $\bmod \Lambda$ turn into triangles in $D^{b}(\bmod \Lambda)$. 


\subsection{Lemma.}

$$
\operatorname{repdim} \Lambda \geq \operatorname{dim} D^{b}(\bmod \Lambda)
$$

Proof. See $[9,12]$.

If we want to use these inequalities to establish lower bounds for the representation dimension, we need to find a method to prove lower bounds for dimensions of triangulated categories or their subcategories. One key ingredient is the ghost lemma.

4.6. Definition. Let $\mathcal{C}^{\prime} \subset \mathcal{C}$ be categories, $f: X \rightarrow Y$ in $\mathcal{C}$ is called $\mathcal{C}^{\prime}$-ghost if any composition $C^{\prime} \rightarrow X \stackrel{f}{\rightarrow} Y$ with $C^{\prime} \in \mathcal{C}^{\prime}$ vanishes.

Here we look at $\langle M\rangle \subset D^{b}(\bmod \Lambda)$.

4.7. Lemma (Ghost lemma). Let $\mathcal{T}$ be a triangulated category, $M \in$ $\mathcal{T}$, and

$$
X_{0} \stackrel{f_{1}}{\longrightarrow} X_{1} \stackrel{f_{2}}{\longrightarrow} X_{2} \stackrel{f_{3}}{\longrightarrow} \ldots \stackrel{f_{n}}{\longrightarrow} X_{n}
$$

a sequence of $\langle M\rangle$-ghosts, such that the composition $f_{1} \cdots f_{n} \neq 0$.

Then $X_{0} \notin\langle M\rangle_{n}$.

Proof. See, for instance, [9].

4.8. Example. Let $\mathcal{T}=D^{b}(\bmod \Lambda), M=\Lambda$, and $X \in \bmod \Lambda$ with pd $X=n$. Then $X \notin\langle M\rangle_{n}$.

Proof. The projective resolution

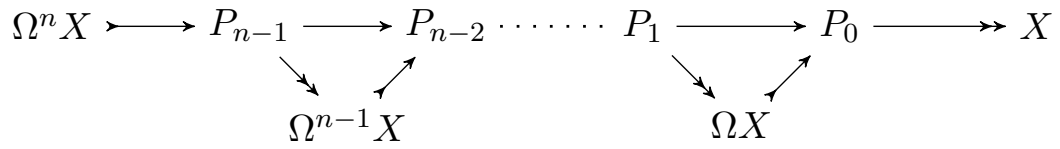

gives rise to a sequence of maps

$$
X \rightarrow \Omega X[1] \rightarrow \cdots \rightarrow \Omega^{n-1} X[n-1] \rightarrow \Omega^{n} X[n]
$$

in $D^{b}(\bmod \Lambda)$. They are all $\langle\Lambda\rangle$-ghosts, and their composition in nonzero. Hence the claim follows from the ghost lemma.

4.9. Remark. For this example it suffices for $\Lambda$ to be a left noetherian ring. 
4.10. Problem. In order to show that $\operatorname{dim} D^{b}(\bmod \Lambda)$ or $\operatorname{dim} \bmod \Lambda$ is big we need to find such a sequence of $\langle M\rangle$-ghosts for an arbitrary $M$.

This problem can be solved "in a different world":

Let $\mathbb{X}$ be a reduced scheme of finite type over $k$. We look at coh $\mathbb{X}$ instead of $\bmod \Lambda$.

4.11. Remark. The easiest (and most important) examples are:

$$
\begin{aligned}
& \mathbb{A}^{n}: \operatorname{coh} \mathbb{A}^{n}=\bmod k\left[x_{1}, \ldots, x_{n}\right] \\
& \mathbb{P}^{n}: \operatorname{coh} \mathbb{P}^{n}=\bmod _{\mathrm{gr}} k\left[x_{0}, \ldots, x_{n}\right] /(\text { modules of finite length })
\end{aligned}
$$

The important difference between $\bmod \Lambda$ and $\operatorname{coh} \mathbb{X}$ is that in the geometric setup we have an extra tool: localization. For any closed point $\mathfrak{p}$ we have the local ring $\mathcal{O}_{\mathfrak{p}}$, and for any $M \in \operatorname{coh} \mathbb{X}$, the corresponding module $M_{\mathfrak{p}} \in \bmod \mathcal{O}_{\mathfrak{p}}$.

We denote by $k_{\mathfrak{p}}=\mathcal{O}_{\mathfrak{p}} / \mathfrak{p}$ the simple sheaf concentrated in point $\mathfrak{p}$. (This is called a "skyscraper sheaf".)

4.12. Lemma. Let $\mathbb{X}$ be a reduced scheme of finite type over $k$, and $M \in \operatorname{coh} \mathbb{X}$. Then $M_{\mathfrak{p}}$ is projective over $\mathcal{O}_{\mathfrak{p}}$ for all $\mathfrak{p}$ in a dense open set of closed points in $\mathbb{X}$.

\subsection{Corollary.}

$$
\operatorname{dim}_{D^{b}(\operatorname{coh} \mathbb{X})}\left\{k_{\mathfrak{p}} \mid \mathfrak{p} \text { a closed point in } \mathbb{X}\right\} \geq \operatorname{dim} \mathbb{X}
$$

Proof. Choose a closed point $\mathfrak{p}$ in an irreducible component of $\mathbb{X}$ which has the same dimension as $\mathbb{X}$, such that $M_{\mathfrak{p}}$ is projective over $\mathcal{O}_{\mathfrak{p}}$. Then

$$
\begin{aligned}
k_{\mathfrak{p}} \in\langle M\rangle_{n} & \Longrightarrow k_{\mathfrak{p}} \in\left\langle M_{\mathfrak{p}}\right\rangle_{n} \subseteq\left\langle\mathcal{O}_{\mathfrak{p}}\right\rangle_{n} \\
\underset{\text { Example }}{\Longrightarrow} & \gtrless \operatorname{pd}_{\mathcal{O}_{\mathfrak{p}}} k_{\mathfrak{p}} \geq \operatorname{dim} \mathbb{X} .
\end{aligned}
$$

Back to finite dimensional algebras. The first examples of algebras of representation dimension strictly bigger then three have been the exterior algebras.

4.14. Theorem (Rouquier). Let $\Lambda$ be the exterior algebra of an $n$ dimensional vector space. Then

$$
\operatorname{repdim} \Lambda=n+1 \text {. }
$$


Vague idea of proof. (see [12] for details) Use Koszul duality

$$
D^{b}(\bmod \Lambda) \stackrel{\operatorname{RHom}(k,-)}{\longrightarrow} D\left(\operatorname{dgmod} k\left[x_{1}, \ldots, x_{n}\right]\right)
$$

and relate the latter category to the derived category of coherent sheaves on $\mathbb{P}^{n-1}$.

Here we want to look at the Beilinson algebras in more detail.

4.15. Theorem. Let $\Lambda$ be the Beilinson algebra (see Example 3.7) $k Q / I$ with

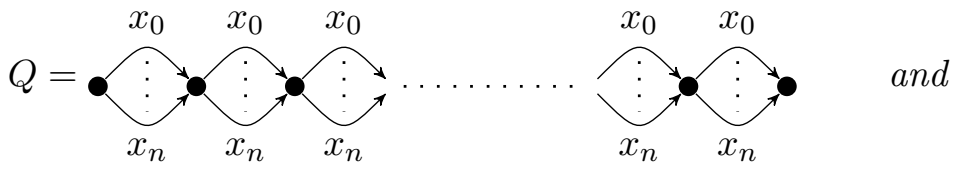

$$
I=\left(x_{i} x_{j}-x_{j} x_{i}\right) .
$$

Then

$$
\operatorname{repdim} \Lambda=n+2
$$

Proof. The sheaf $T=\mathcal{O} \oplus \mathcal{O}(1) \cdots \oplus \mathcal{O}(n)$ is a tilting bundle in coh $\mathbb{P}^{n}$ with $\operatorname{End}(T)=\Lambda$. Hence it induces a derived equivalence

$$
D^{b}\left(\operatorname{coh} \mathbb{P}^{n}\right) \stackrel{\operatorname{RHom}(T,-)}{\longrightarrow} D^{b}(\bmod \Lambda) .
$$

Since $\operatorname{Ext}_{\mathbb{P} n}^{i}\left(\mathcal{O}(j), k_{\mathfrak{p}}\right)=0$ for $i \geq 1$, for any $j$, and any closed point $\mathfrak{p}$, the functor $\operatorname{RHom}(T,-)$ maps $\left\{k_{\mathfrak{p}} \mid \mathfrak{p}\right.$ closed point $\}$ to $\bmod \Lambda$. Hence

$$
\begin{aligned}
& \operatorname{dim}_{D^{b}(\bmod \Lambda)} \bmod \Lambda \geq \operatorname{dim}_{D^{b}(\bmod \Lambda)} \operatorname{RHom}\left(T,\left\{k_{\mathfrak{p}} \mid \mathfrak{p} \text { closed point }\right\}\right) \\
&=\operatorname{dim}_{D^{b}(\operatorname{coh} \mathbb{X})}\left\{k_{\mathfrak{p}} \mid \mathfrak{p} \text { closed point }\right\} \\
& \geq n \\
& \text { Corollary } 4.13
\end{aligned}
$$

Now repdim $\Lambda \geq n+2$ by Lemma 4.4. We have seen $\operatorname{repdim} \Lambda \leq n+2$ in Example 3.7.

4.16. Remark. The proof above (showing that repdim $\Lambda \geq n+2$ ) works for any algebra $\Lambda$ that comes up as the endomorphism ring of a tilting object in $\operatorname{coh} \mathbb{X}$, where $\mathbb{X}$ is a reduced scheme of dimension $n$. 
4.17. Example. For $\mathbb{X}=\underbrace{\mathbb{P}^{1} \times \cdots \times \mathbb{P}^{1}}_{n \text { copies }}$ there is a tilting bundle

$$
T=\oplus_{i \in\{0,1\}^{n}} \mathcal{O}\left(i_{1}\right) \otimes \cdots \otimes \mathcal{O}\left(i_{n}\right) .
$$

By Remark 4.16 the endomorphism ring of $T$ has representation dimension at least $n+2$. Using Iyama's upper bound (Proposition 3.4) one easily sees that $\operatorname{repdim} \Lambda=n+2$.

The algebras arising here have the following shape:

$n=1: \operatorname{End}(T)=k[\bullet \longrightarrow \bullet$

$n=2: \operatorname{End}(T)=k Q / I$ with

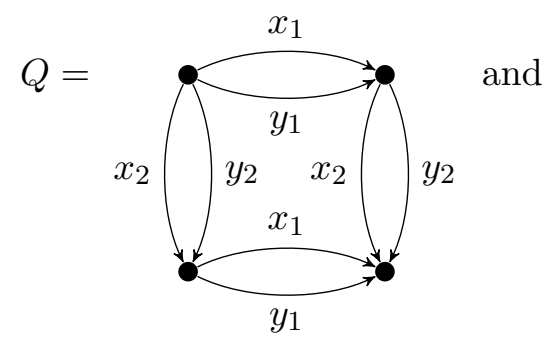

$$
I=\left(x_{1} x_{2}-x_{2} x_{1}, x_{1} y_{2}-y_{2} x_{1}, y_{1} x_{2}-x_{2} y_{1}, y_{1} y_{2}-y_{2} y_{1}\right) .
$$

$n=3: \operatorname{End}(T)=k Q / I$ with

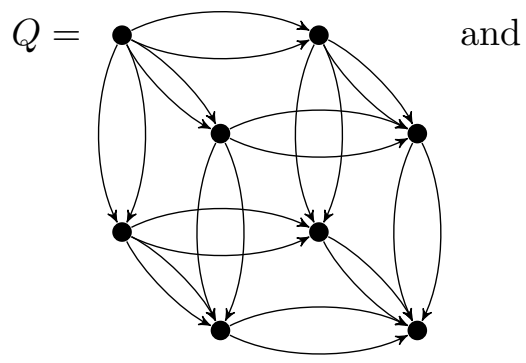

$$
I=(\text { similar commutation relations }) .
$$

and similar hypercubes with commutation relations for larger $n$.

We conclude by giving two more general results giving lower bounds for the representation dimension by relating the module category to some commutative setup: 
Setup for the lattice theorem: Let $R$ be a finitely generated commutative algebra over $k$ without zero divisors, and let $\Lambda$ be a finite dimensional $k$-algebra. Let $L$ be a $\Lambda \otimes_{k} R$-lattice (that is a bimodule, which is projective over $R$ ). Then the functor

$$
L \otimes_{R}-\text { : finite length modules over } R \longrightarrow \bmod \Lambda
$$

is exact, and hence induces maps

$$
\left(L \otimes_{R}-\right)_{\operatorname{Ext}^{d}}: \operatorname{Ext}_{R}^{d}(X, Y) \longrightarrow \operatorname{Ext}_{\Lambda}^{d}\left(L \otimes_{R} X, L \otimes_{R} Y\right)
$$

for $R$-modules $X$ and $Y$ of finite length.

4.18. Theorem (Lattice theorem - see [10]). Let $\Lambda, R$ and $L$ be as above, and $d \in \mathbb{N}$. Assume

$$
\left(L \otimes_{R}-\right)_{\operatorname{Ext}^{d}}\left(\operatorname{Ext}_{R}^{d}(R / \mathfrak{p}, R / \mathfrak{p})\right) \neq 0
$$

for all $\mathfrak{p}$ in a dense subset of maximal ideals of $R$. Then

$$
\operatorname{repdim} \Lambda \geq d+2 \text {. }
$$

4.19. Theorem (Bergh [4]). Assume $\Lambda$ is self-injective, and the even Hochschild cohomology ring satisfies a finite generation hypothesis (see [4]). Then

$$
\text { repdim } \Lambda \geq \text { Krull.dim } \mathrm{HH}^{2 *}(\Lambda)+1 .
$$

(Here Krull.dim $\mathrm{HH}^{2 *}(\Lambda)$ denotes the Krull dimension of the (commutative) even Hochschild cohomology ring.)

\section{References}

[1] Maurice Auslander, Representation dimension of Artin algebras, Queen Mary College Mathematics Notes, 1971, republished in [2].

[2] _ Selected works of Maurice Auslander. Part 1, American Mathematical Society, Providence, RI, 1999, Edited and with a foreword by Idun Reiten, Sverre O. Smalø, and Øyvind Solberg.

[3] Maurice Auslander, Idun Reiten, and Sverre O. Smalø, Representation theory of Artin algebras, Cambridge Studies in Advanced Mathematics, vol. 36, Cambridge University Press, Cambridge, 1997, Corrected reprint of the 1995 original.

[4] Petter Andreas Bergh, Representation dimension and finitely generated cohomology, Adv. Math. 219 (2008), no. 1, 389-400.

[5] Karin Erdmann, Thorsten Holm, Osamu Iyama, and Jan Schröer, Radical embeddings and representation dimension, Adv. Math. 185 (2004), no. 1, 159-177. 
[6] Kiyoshi Igusa and Gordana Todorov, On the finitistic global dimension conjecture for Artin algebras, Representations of algebras and related topics, Fields Inst. Commun., vol. 45, Amer. Math. Soc., Providence, RI, 2005, pp. 201-204.

[7] Osamu Iyama, Rejective subcategories of artin algebras and orders, preprint, arXiv:math.RT/0311281.

[8] , Finiteness of representation dimension, Proc. Amer. Math. Soc. 131 (2003), no. 4, 1011-1014.

[9] Henning Krause and Dirk Kussin, Rouquier's theorem on representation dimension, Trends in representation theory of algebras and related topics, Contemp. Math., vol. 406, Amer. Math. Soc., Providence, RI, 2006, pp. 95103.

[10] Steffen Oppermann, Lower bounds for Auslander's representation dimension, Duke Math. J. 148 (2009), no. 2, 211-249 .

[11] Claus Michael Ringel, The torsionless modules of an artin algebra, 2008.

[12] Raphaël Rouquier, Representation dimension of exterior algebras, Invent. Math. 165 (2006), no. 2, 357-367.

[13] Dimensions of triangulated categories, J. K-Theory 1 (2008), no. 2, $193-256$. 